\title{
THEORETICAL STUDY OF A TWIN-TUBE MAGNETORHEOLOGICAL DAMPER CONCEPT
}

\author{
JANUSZ GOEDASZ \\ Technical Center Krakow, BWI Group, Kraków, Poland and \\ Cracow University of Technology, Department of Control and Information Technology, Kraków, Poland \\ e-mail: janusz.goldasz@bwigroup.com
}

\begin{abstract}
In this study, the author presents a theoretical model of a semi-active magnetorheological (MR) twin-tube damper concept. The model relies on geometric variables and material properties and can be used in engineering and research studies on damper structures. Other non-linear characteristics, namely, the fluid chamber compressibility, fluid inertia, cylinder elasticity, friction, one-way check valves are included into the model as well. The author studies the performance of the damper model as design variables are varied, and the results are analysed and discussed.
\end{abstract}

Keywords: MR damper, twin-tube damper concept, lumped parameter model

\section{Introduction}

Magnetorheological (MR) fluids have always been attractive to engineers and researchers within the automotive industry. The material adapts to changing external conditions within milliseconds. Automotive (vehicle) dampers utilizing MR fluids are now found in a number of semi-active platforms in vehicles. In the industry, the monotube damper configuration (de Carbon, 1952 ) is the most common structure of a flow-mode MR damper. The cylinder tube houses the floating piston (gas cup) separating the fluid from the gas-filled chamber. The piston divides the MR fluid volume into the compression chamber (fluid volume between the floating piston and the main piston assembly) and the rebound chamber (fluid volume between the rod guide and the main piston). The piston assembly contains an annular gap to permit the fluid to flow between the chambers and secondary flow paths (bypasses) for tuning the MR damper low-speed performance. In a typical MR damper, the rod is attached to the vehicle body and the cylinder to the wheel hub. The relative motion of the wheel and the body drives the fluid flow between the chambers through the annulus in the piston. The design has been a natural choice for MR applications due to its simplicity, however, high operating pressures and packaging limit its scope. Moreover, manufacturing issues due to high surface finish requirements of the cylinder tube are a factor here, too. Also, gas high pressures in monotube dampers would translate into rod guide friction well above that of twintube hardware. Therefore, the research on other structures of MR dampers continues (Poynor, 2001). A standard twin-tube damper features concentric cylinder tubes. The inner cylinder houses a piston valve for controlling the flow between the adjacent fluid chambers and a base (foot) valve for regulating the flow between the fluid chamber below the piston in the inner cylinder and a reservoir (fluid volume contained between the outer tube and the inner one). The reservoir is partially filled with oil to accommodate volume changes due to rod displacement. The dampers work at a lower gas pressure, but only upright positions are possible in vehicles, and they incorporate more valves. However, research efforts on MR twin-tube structures have not fully succeeded. Two studies focused on a twin-tube structure of an MR damper in which the MR control valve was located in the piston inside the inner cylinder 
(Poynor, 2001; Jensen et al., 2001). In the design of Jensen et al. (2001), a standard base valve was used for controlling the MR fluid flow into the outer reservoir. The damper structure, however, might suffer from hydraulic imbalance (a common problem affecting twintube dampers), and the range of damping forces that can be achieved with this design could be limited. The imbalance phenomenon occurs when the damper is in compression and the pressure drop across the piston is larger than the pressure drop across the base valve. As a result, most of the fluid volume is pushed through the base valve causing lags in the chamber above the piston. Another study revealed a twin-tube damper in which the MR valve regulates the fluid flow from the upper chamber above the piston into the reservoir volume between the cylinders (Oakley, 2006). Two one-way check valves are used for directing the flow between the fluid chambers. Another feature of this concept is its ability to tune its non-energized condition with passive valves. Apparently, there is no published research on the twin-tube design of Oakley (2006) related to its performance. The proposed model fulfills this gap. Briefly, the generic goal of this study was to provide a lumped parameter model of a twin-tube MR damper for component as well as vehicle level analyses. The task is complicated - damper and flow channel geometry, magnetic field induced yield stress and resistance-to-flow build-up, fluid compliance, cavitation, friction, gas absorption, etc. have been among the contributors to the force output of MR dampers (Hong et al., 2006). At the same time, vehicle dampers have been a subject of intensive modelling work. In the past, researchers developed various models of dampers to copy their non-linear characteristics. For example, Lang (1977) as well as Segel and Lang (1981) developed a math model of a twintube automotive damper and concluded the observed hysteretic behavior was due to the compressibility of the fluid, cylinder tube elasticity and cavitation. The models of Lang (1977), Segel and Lang (1981) remain the key work on conventional dampers operating at high frequencies. Also, Lee (1997) obtained a complex model of a monotube vehicle suspension damper. The model included compressibility of fluid dampers, floating gas cup inertia and first-order heat transfer effects in addition to a deflected disc piston model. Also, Mollica (1997) proposed a non-linear model of a monotube damper using bond graph techniques. The model of Mollica incorporates friction elements, fluid compressibility, gas, leakage and hydraulic resistance components in the piston (Mollica, 1997). Those studies were a basis for developing the lumped parameter model described in detail below. Specifically, the goal was to obtain a damper model capable of copying the performance characteristics of twin-tube MR dampers and important phenomena occurring inside the device as well as the operational logic of the damper. Also, fluid compressibility effects and fluid inertia are modeled, and their influence on the damping force output of an MR damper is analysed for a selected configuration.

\section{Modelling}

The MR twin-tube damper concept is illustrated in Fig. 1. The inner tube houses the piston separating the fluid volume into the rebound (upper) chamber volume and the compression (lower) chamber volume. The damper is driven by the displacement (velocity) input $x_{p}\left(v_{p}\right)$ applied to the rod. MR valve (1) controls the fluid flow between rebound and reservoir chambers. The flow rate through the MR valve is $Q_{v, 1}$. The flow through the piston $Q_{v, 2}$ is controlled by check valve (2). The valve allows flow in one direction only, from chamber (2) (compression) into chamber (1) (rebound). The flow between chambers (3) (reservoir) and (2) (compression) is controlled by one-way valve (3). This valve allows flow from chamber (3) (reservoir) into (2) (compression). Both valves are schematically shown in Fig. 1 - they may take the form of a standard deflected disc stack assembly or a preloaded spring and plate. The flow rate through check valve (3) is $Q_{v, 3}$. The reservoir contains MR fluid and pressurised gas. The fluid rheology in the annulus is controlled by the magnetic field $H$ due to the current $I_{c}$ in the coil of the piston 
core. The fluid is described by the yield stress $\tau_{0}$, viscosity $\mu$, density $\rho$, and bulk modulus $B_{f}$. The MR annulus height is $h$, and its cross-section area $A_{g} . L_{g}$ is the annular length, and the active section length (magnetic poles) is $L_{a}\left(L_{a}<L_{g}\right)$. In rebound (see Fig. 1), the rod moves out of the damper. The flow is through valves (1) and (3), and there is no flow through valve (2); the flow through MR valve (1) is uni-directional. In compression, the rod would move into the damper. Flow through check valve (3) would be prevented, and it would occur through valves (1) and (2). In the sections that follow below, the author discusses the key phenomena occurring in the damper and outside of the MR valve.

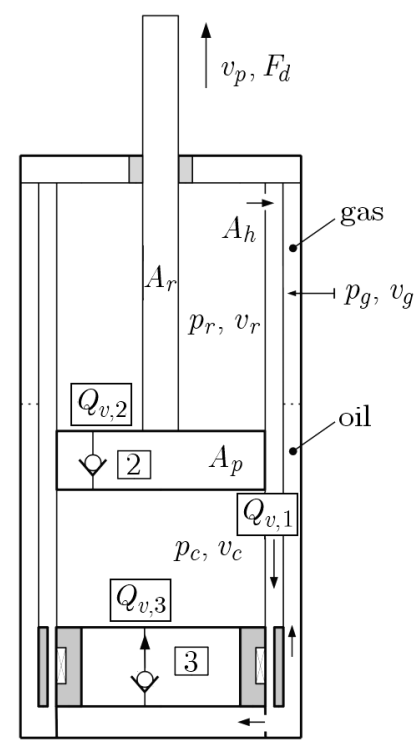

Fig. 1. MR twin-tube damper: internal MR valve

\subsection{Damper model}

Consider the damper model in Fig. 1. With the inertia of the lumped mass of fluid in the MR valve annulus, the force balance equation is (Gołdasz and Sapiński, 2013)

$$
\dot{Q}_{v, 1}=\frac{A_{g}}{\rho L_{g}}\left(P_{r}-P_{g}-\Delta p_{a}-\Delta P_{H}\right)
$$

where $\Delta p_{a}$ is the field-induced pressure drop along the annular gap, and $\Delta P_{H}$ denotes losses at the holes in the inner cylinder. The term $\Delta p_{a}$ is discussed in detail in Section 2.2. Also, fluid continuity expressions for the pressures above and below the piston are

$$
\begin{aligned}
& \dot{P}_{r}=\beta\left(P_{r}\right) \frac{\left(A_{p}-A_{r}\right) v_{p}-\left(Q_{v, 1}+Q_{v, 2}\right)}{V_{r, 0}-\left(A_{p}-A_{r}\right) x_{p}} \\
& \dot{P}_{c}=\beta\left(P_{c}\right) \frac{-A_{p} v_{p}+\left(Q_{v, 2}+Q_{v, 3}\right)}{V_{c, 0}+A_{p} x_{p}}
\end{aligned}
$$

where $\beta(P)$ refers to the combined bulk modulus due to fluid compressibility and cylinder compliance, whereas $V_{r, 0}$ and $V_{c, 0}$ are midstroke fluid chamber volumes. Gas pressure in the reservoir $P_{g}$ can be expressed assuming the adiabatic process, i.e. without heat transfer between the damper and the environment

$$
P_{g}=P_{g, 0}\left(\frac{V_{g, 0}}{V_{g, 0}-\int\left(Q_{v, 1}-Q_{v, 3}\right) d t}\right)^{n}
$$


In the above equation, $P_{g, 0}$ and $V_{g, 0}$ are the initial gas pressure and volume, respectively, and $n$ is the adiabatic gas constant. Also in this analysis, the effects of wall expansion with pressure are combined with the influence of fluid bulk modulus via the relationship

$$
\frac{1}{\beta}=\frac{1}{\beta_{f}}+\frac{1}{\beta_{s}}
$$

where the variation of the fluid bulk modulus with pressure can be as

$$
\beta_{f}(P)=\beta_{0} \frac{1+\alpha\left(\frac{P_{a}}{P_{a}+P}\right)^{\frac{1}{n}}}{1+\alpha \frac{P_{a}^{\frac{1}{n}}}{n\left(P_{a}+P\right)^{\frac{1+n}{n}}}}
$$

Equation (2.5) reveals the bulk modulus variation with pressure of the mixture of the fluid and non-dissolved air (Manring, 2005). $\beta_{0}$ is the pure fluid bulk modulus, $P_{a}$ refers to the atmospheric (or reference) pressure, and $\alpha$ denotes the relative gas content. The compliance of the steel cylinder $\beta_{s}$ is (Mollica, 1997)

$$
\frac{1}{\beta_{s}}=\frac{2}{E_{s}}\left(\nu+\frac{D_{o}^{2}+D_{p}^{2}}{D_{o}^{2}-D_{p}^{2}}\right)
$$

where $E_{s}$ is Young modulus (steel), $\nu$ - Poisson's coefficient, $D_{o}$ - outer diameter of the cylinder. Cavitation effects are simply modeled by imposing a constraint on the pressures $P_{r}$ and $P_{c}$, $P_{r} \geqslant P_{v}$ and $P_{c} \geqslant P_{v}$. Also, the pressure drop at the holes $\Delta P_{H}$ in the inner cylinder is

$$
\Delta P_{H}=\rho \frac{Q_{v, 1}^{2}}{2\left(C_{H} A_{H}\right)^{2}}
$$

where $C_{H}$ is the discharge coefficient and $A_{o}$ cross-sectional area of the holes. Using the one-way valve in the piston, the piston flow rate $Q_{v, 2}$ can be

$$
Q_{v, 2}= \begin{cases}C_{2} A_{2} \sqrt{2 \frac{\left|P_{r}-P_{c}\right|}{\rho}} & P_{r}-P_{c}<0 \\ 0 & P_{r}-P_{c} \geqslant 0\end{cases}
$$

Similarly, the flow rate $Q_{v, 3}$ through check valve (3) is

$$
Q_{v, 3}= \begin{cases}C_{3} A_{3} \sqrt{2 \frac{\left|P_{c}-P_{g}\right|}{\rho}} & P_{c}-P_{g}<0 \\ 0 & P_{c}-P_{g} \geqslant 0\end{cases}
$$

The check valves are assumed to open with no delay. Considering forces on the piston, the damping force $F_{d}$ including friction $F_{f}$ becomes

$$
F_{d}=\left(A_{p}-A_{r}\right) P_{r}-A_{p} P_{c}+F_{f}\left(\operatorname{sgn}\left(v_{p}\right)\right)
$$

To summarize, equations from (2.1) to (2.10) form a set of expressions for simulating the output of a twin-tube MR damper. 


\subsection{MR valve model}

This Section shows the application of a biplastic Bingham scheme for deriving the pressure vs. flow rate characteristics of an MR valve model. The MR valve (annulus) contains a parallel flux bypass feature. The flux bypass often takes the form of a slot feature on either surface constituting the annulus. Due to the increased (local) height of the annulus, it is characterized by a region of low flux density (yield stress) (Gołdasz and Sapiński, 2012) where the MR fluid is allowed to flow through the flux bypass section at a lower breakaway pressure drop than in the other portion of the flow channel. As a result, low forces are achieved at near-zero flow rates through the MR piston. Medium and high flow rate performance is not affected. Application of the bi-plastic scheme is based on the assumption that the dual behavior can be described with the artificial material model of parameters related to both material properties of the MR (Bingham) fluid and the piston geometry. By expressing the pressure drop $\Delta p_{a}$ across the control valve in terms of the dimensionless pressure number $G$ and the plasticity $S$, the equation linking the term $\Delta p_{a}$ with the flow rate through the MR valve $Q_{v, 1}$ is (Gołdasz and Sapiński, 2012)

$$
\begin{aligned}
& \Delta p_{a}=\frac{2 \tau_{2} L_{a}}{h} G(S)+C \frac{\rho Q_{v, 1}^{2}}{A_{g}^{2}}=\frac{2 \tau_{0} L_{a}}{h[1-\gamma(1-\delta)]} G(S)+C \frac{\rho Q_{v, 1}^{2}}{A_{g}^{2}} \\
& G=-\frac{h \Delta p_{a}}{2 L_{a} \tau_{2}} \quad S=\frac{12 \mu Q_{v, 1}}{w h^{2} \tau_{2}}
\end{aligned}
$$

In equation (2.11), high velocity losses are accounted for in the model in quadratic form, and the tuning coefficient $C$ captures the effects of the fluid entry and exit, flow development, turbulent losses, etc. The parameters $\gamma$ and $\delta$ refer to the slope of the damper force (pressure) variation against velocity (flow rate) and the interception force in the pre-yield region, and $\tau_{2}$ is the bi-plastic material yield stress. The pre-yield viscosity (slope) $\mu_{r}$ is related to the material viscosity $\mu$ via $\gamma=\mu / \mu_{r}$, and the yield stress $\tau_{2}$ is linked to the yield stress $\tau_{0}$ through the equation $\tau_{0}=\tau_{2}[1-\gamma(1-\delta)]$. At $\gamma \rightarrow \infty$ and $\delta \rightarrow 1$, the model would reduce to that of classic Bingham's.

The bi-plastic model was studied by various authors (Gołdasz and Sapiński, 2012, 2013; Dimock et al., 2002). For example, Gołdasz and Sapiński (2012) analyzed the performance of a dual coil MR piston with the flux bypass feature and extracted non-dimensional parameters for it. The authors concluded that the non-dimensional viscosity $\gamma$ was relatively invariant of the magnetic field, whereas the yield stress parameter $\delta$ varied with the current level (or flux density). The model allows for separating the flow regime into two distinct flow regimes with the threshold plasticity $S_{0}=\gamma\left(2-3 \delta+\delta^{3}\right)$. Briefly, the pre-yield (bypass) regime is characterized by the plasticity number $S<S_{0}$ and the post-yield regime by $S \geqslant S_{0}$. In the model, the post-yield relationship between the pressure drop and the flow rate through the annulus for $\left(S \geqslant S_{0}\right.$ and $G \geqslant 1)$ is

$$
G=\frac{1}{6}[3(1-\gamma(1-\delta))+S]\left[2 \cos \left(\frac{1}{3} \arctan 2(y, x)\right)+1\right]
$$

where

$$
\begin{array}{rlrl}
y & =12 \sqrt{-81 b^{2}+12 b a^{3}} & x & =-108 b+8 a^{3} \\
a & =\frac{3}{2}(1-\gamma(1-\delta))+\frac{1}{2} S & b & =\frac{1}{2}\left(1-\gamma\left(1-\delta^{3}\right)\right)
\end{array}
$$

In the pre-yield flow regime, $S<S_{0}$, the material behavior is governed by the modified Bingham plastic formula

$$
G=\delta \frac{1}{6}\left(\frac{S}{\delta \gamma}+3\right)\left[2 \cos \left(\frac{1}{3} \arctan 2\left(y^{\prime}, x^{\prime}\right)\right)+1\right]
$$


where

$$
\begin{aligned}
& x^{\prime}=-27+27 \frac{S}{\gamma \delta}+9\left(\frac{S}{\gamma \delta}\right)^{2}+\left(\frac{S}{\gamma \delta}\right)^{3} \\
& y^{\prime}=6 \sqrt{3} \sqrt{27 \frac{S}{\gamma \delta}+9\left(\frac{S}{\gamma \delta}\right)^{2}+\left(\frac{S}{\gamma \delta}\right)^{3}}
\end{aligned}
$$

To summarize, equation (2.11) accompanied by equations (2.14) and (2.15) allow for calculation of the pressure drop $\Delta p_{a}$ across the energized annulus.

\section{Simulations}

The simulations involved the MR twin-tube damper model subjected to a displacement waveform at the rod as in Fig. 1 and used the data in Table 1 . The friction estimate $F_{f}$ of $70 \mathrm{~N}$ has been obtained from a real damper; the gas pressure $P_{g, 0}$ is equal to $0.8 \mathrm{MPa}$, and the adiabatic constant 1.4. The MR fluid bulk modulus $\beta_{f}$ is $1500 \mathrm{MPa}$, the density $\rho$ is $2.68 \mathrm{~g} / \mathrm{cc}$, and its air contents $\alpha$ equal to 0.001 . The viscosity of the fluid $\mu$ is $62 \mathrm{cP}$ at the temperature $T_{a}$ of $30^{\circ} \mathrm{C}-$ see Fig. 2. The steel modulus of elasticity $E_{s}$ is $2.1 \cdot 10^{5} \mathrm{MPa}$, and the Poisson coefficient equals to 0.29 .

Table 1. Twin-tube damper model inputs

\begin{tabular}{|l|l|c|}
\hline \multicolumn{1}{|c|}{ Symbol } & \multicolumn{1}{|c|}{ Description } & Value \\
\hline \hline$L_{r, 0}$ & Initial rebound chamber length, $[\mathrm{mm}]$ & 150 \\
\hline$L_{c, 0}$ & Initial compression chamber length, $[\mathrm{mm}]$ & 150 \\
\hline$A_{e f f}=A p-A_{r}$ & Upper chamber cross-section area, $\left[\mathrm{mm}^{2}\right]$ & 683.48 \\
\hline$A_{p}$ & Cylinder cross-section area, $\left[\mathrm{mm}^{2}\right]$ & 804.24 \\
\hline$V_{r, 0}$ & Initial rebound chamber volume, $\left[\mathrm{mm}^{3}\right]$ & $1.206 \cdot 10^{5}$ \\
\hline$V_{c, 0}$ & Initial compression chamber volume, $\left[\mathrm{mm}^{3}\right]$ & $1.025 \cdot 10^{5}$ \\
\hline$V_{g, 0}$ & Initial gas chamber volume, $\left[\mathrm{mm}^{3}\right]$ & $0.861 \cdot 10^{5}$ \\
\hline$A_{2}, A_{3}$ & Check valve flow areas, $\left[\mathrm{mm}^{2}\right]$ & 220 \\
\hline$C_{2}, C_{3}, C_{H}$ & Discharge coefficients, $[-]$ & 0.7 \\
\hline$A_{H}$ & Cylinder holes area, $\left[\mathrm{mm}^{2}\right]$ & 301 \\
\hline$t_{w}$ & Cylinder wall thickness, $[\mathrm{mm}]$ & 1.8 \\
\hline$L_{a}$ & Active length, $[\mathrm{mm}]$ & 25.8 \\
\hline$L$ & Annulus length, $[\mathrm{mm}]$ & 37 \\
\hline$h$ & Annulus height, $[\mathrm{mm}]$ & 0.89 \\
\hline$w$ & Mean circumferential width, $[\mathrm{mm}]$ & 88.60 \\
\hline$C$ & Flow coefficient, $[-]$ & 0.1 \\
\hline
\end{tabular}

The piston parameters, the yield stress ratio and the viscosity ratio variation with current, respectively, copy the dual-coil assembly by Gołdasz and Sapiński (2012). In the study, the two parameters $\gamma$ and $\delta$ are identified from real piston performance data. The identified viscosity ratio $\gamma$ varied from 0.0175 at the coil current $I_{c}$ of $1 \mathrm{~A}$ through 0.0167 at $3 \mathrm{~A}$ to 0.0149 at the maximum coil current level of $5 \mathrm{~A}$. The yield stress ratio varied from $0.179\left(I_{c}=1 \mathrm{~A}\right)$ through $0.363\left(I_{c}=3 \mathrm{~A}\right)$ to $0.492\left(I_{c}=5 \mathrm{~A}\right)$. Here, the MR piston is simply described by the steady-state pressure vs. flow rate characteristics in Fig. 3. The $\Delta p_{a}-Q_{v, 1}$ characteristics in Fig. 3 are based on the geometry and material properties, and then input into the Simulink model. The fluid data are in Fig. 2; $B$ - magnetic flux density, $H$ - field strength. The results given by equations (2.2) through (2.10) are presented in Figs. 4 through 7 . Briefly, the model 


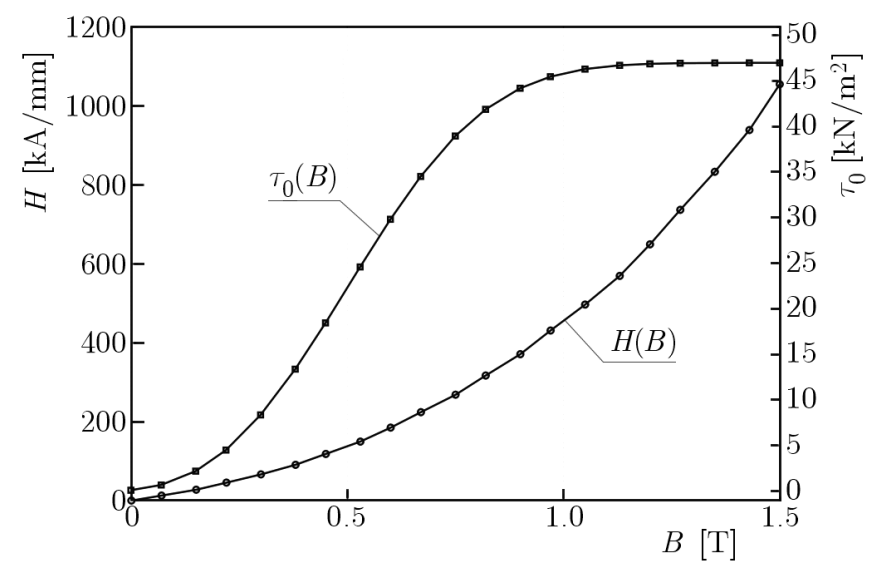

Fig. 2. MR fluid characteristics: $B-H, \tau_{0}-B$ (Gołdasz and Sapiński, 2012)

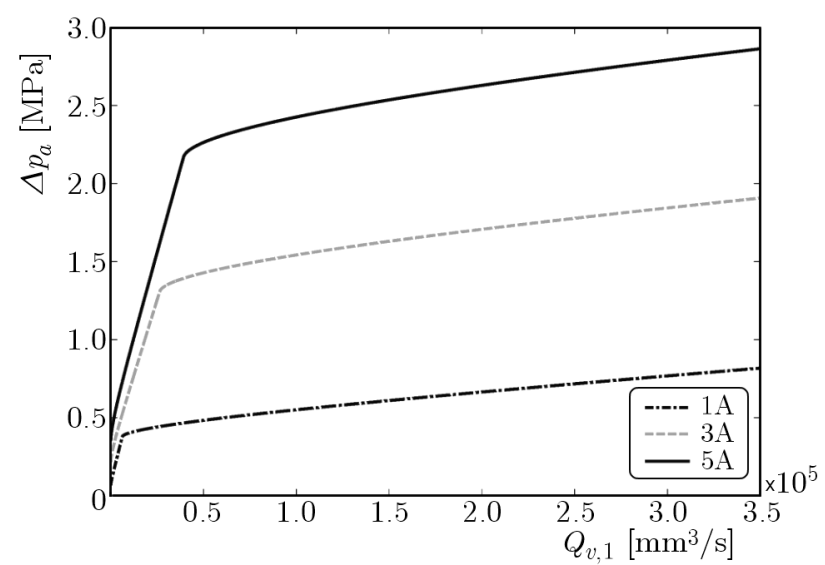

Fig. 3. MR piston steady-state characteristics: $\Delta p_{a}$ vs. $Q_{v, 1}$
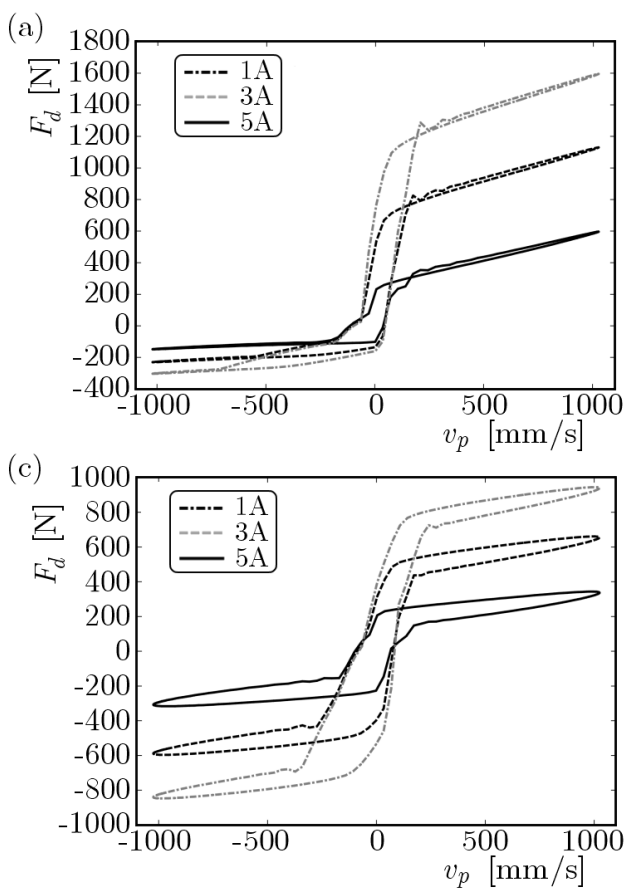

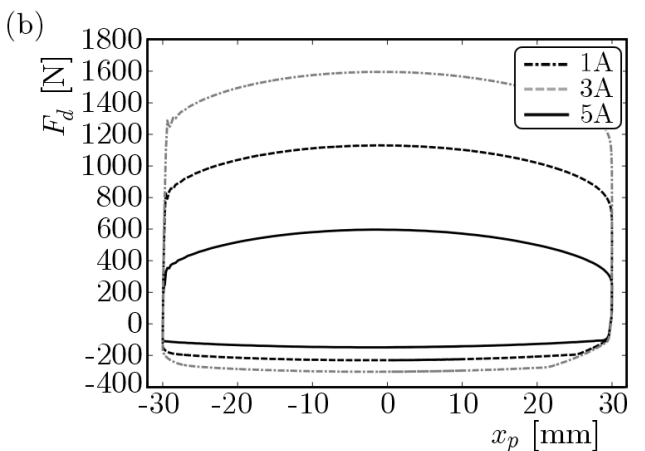

(d)

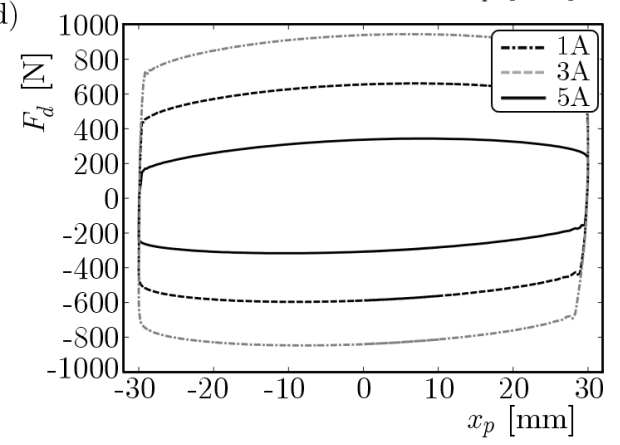

Fig. 4. Influence of rod size on the damping force; $X_{p}=30 \mathrm{~mm}, V_{p}=1024 \mathrm{~mm} / \mathrm{s}$ 

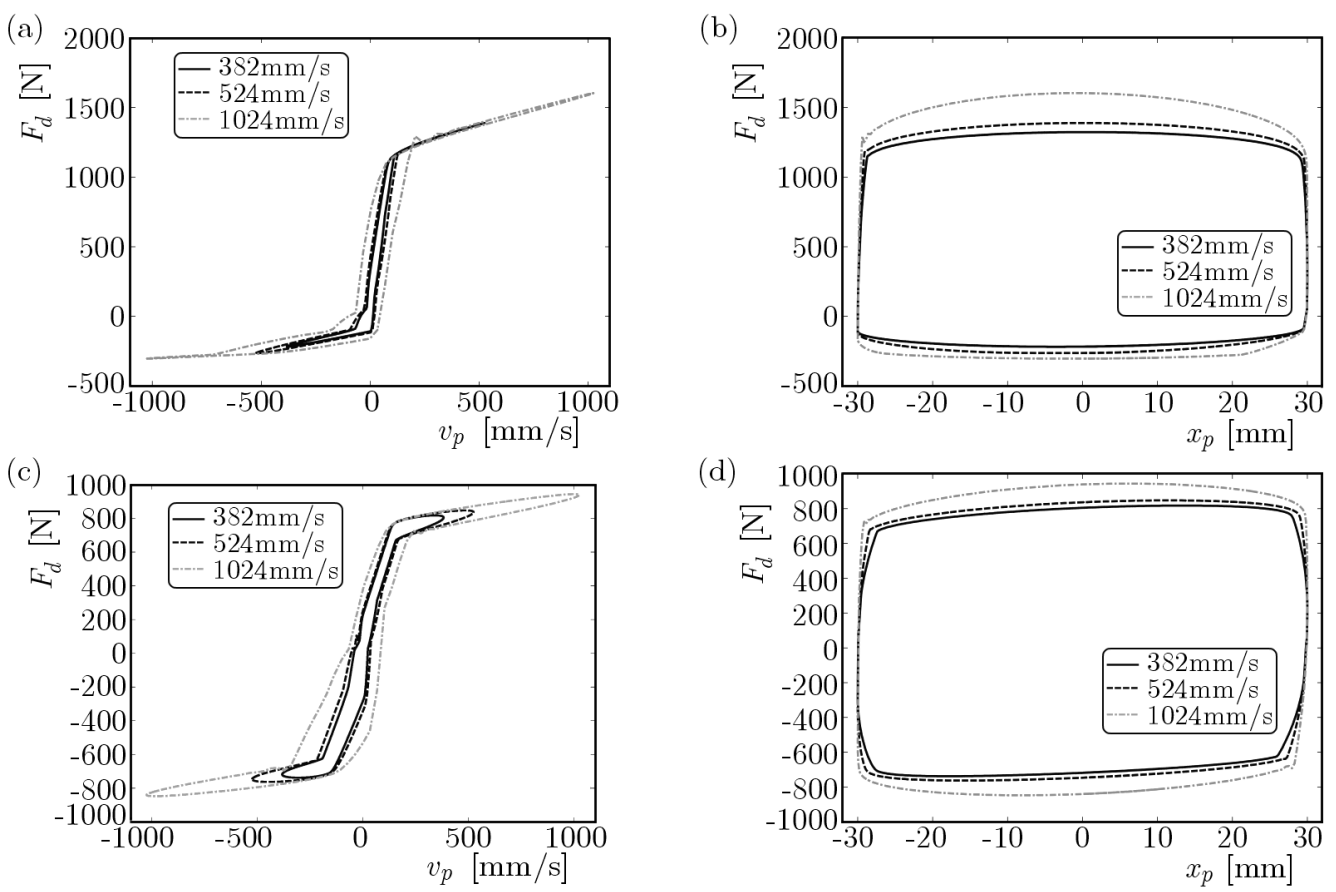

Fig. 5. Graphs of force-displacement and force-velocity; $X_{p}=30 \mathrm{~mm}, I_{c}=5 \mathrm{~A}$
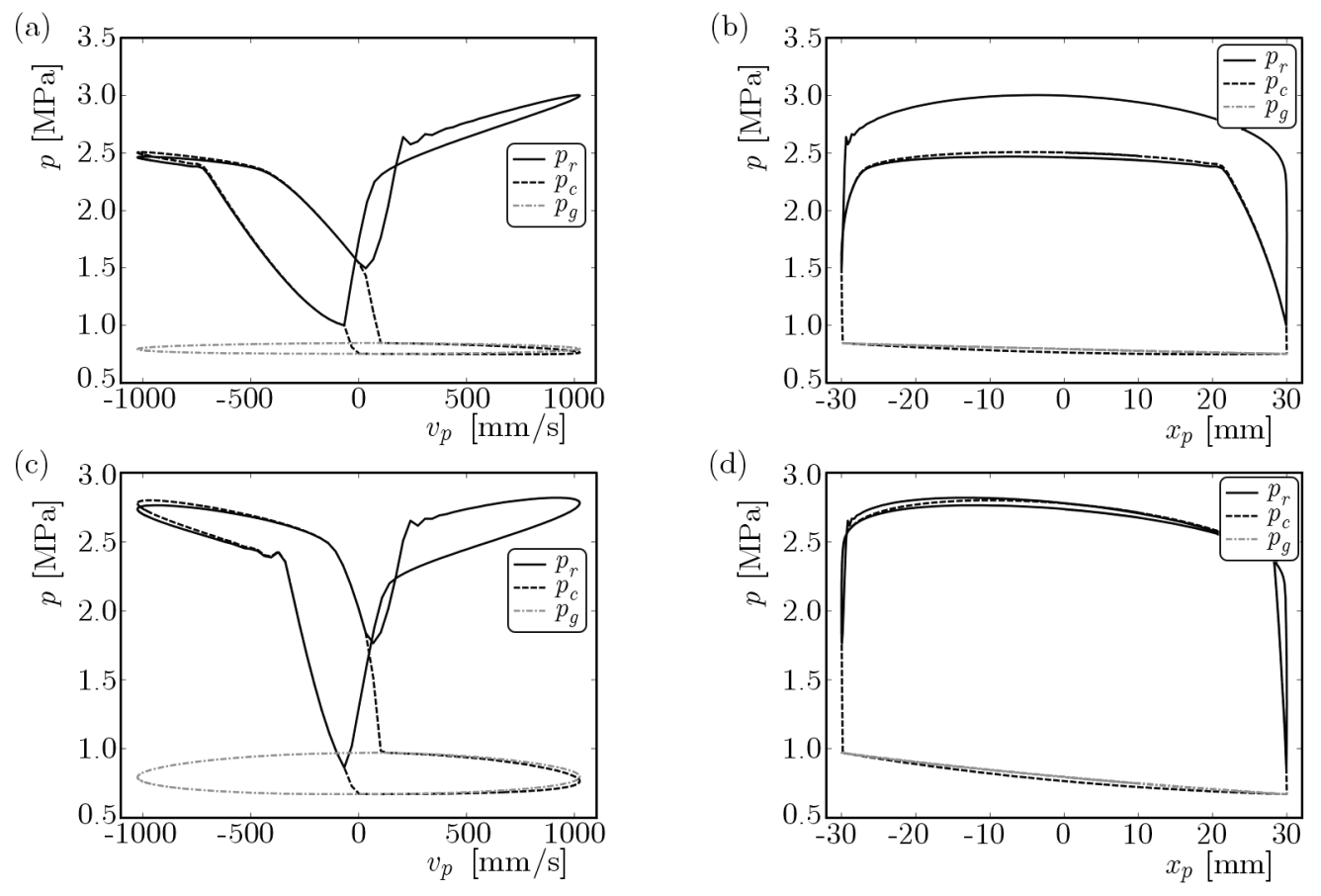

Fig. 6. Graphs of pressure-displacement and pressure-velocity; $X_{p}=30 \mathrm{~mm}, I_{c}=5 \mathrm{~A}$

is subjected to the displacement $x_{p}(t)=X_{p} \sin \omega t$ applied to the rod. The results are shown as force-velocity and force-displacement loops. In the simulations, the effects of velocity, coil current and rod size on the damping force output are examined. Specifically, Fig. 4 shows the impact the rod diameter (area) has on the damper force. As seen in Figs. 4a through 4d, smaller rod sizes $\left(D_{p}=12.4 \mathrm{~mm}\right)$ contribute to major asymmetry in the damping force. The rebound-to-compression ratio (asymmetry ratio) for the damping force is above 5:1 at the peak velocity of $1024 \mathrm{~mm} / \mathrm{s}$. In the cases shown, the rebound forces decreased when the piston diameter increased 

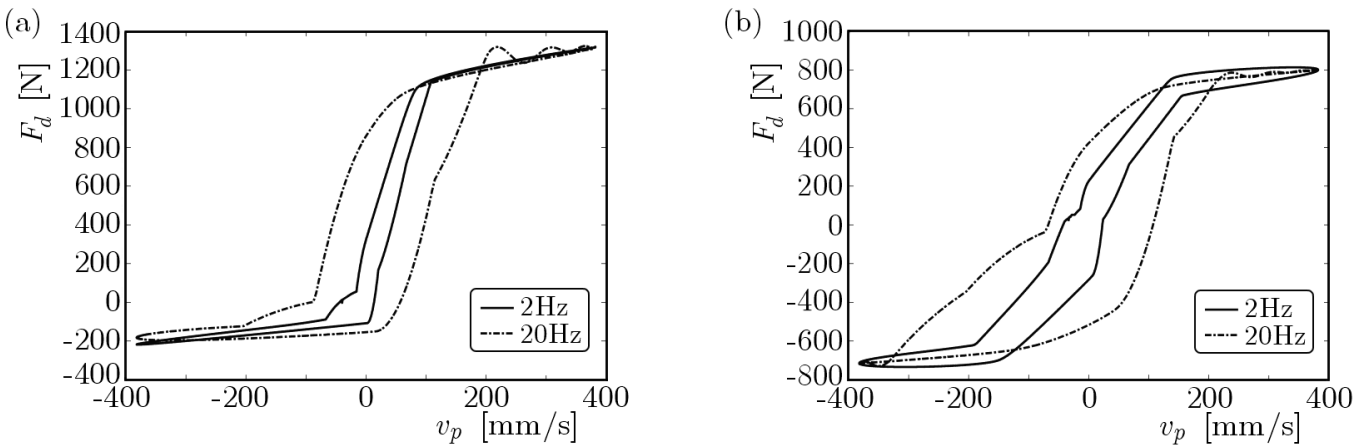

Fig. 7. Influence of the frequency; $V_{p}=382 \mathrm{~mm} / \mathrm{s}$

up to $22 \mathrm{~mm}$. The asymmetry decreased at the expense of rebound forces. It can be shown that as the piston rod is in compression, check valve (2) in the piston is opened, and check valve (3) in the base valve is closed, so that the annular flow rate is related to the rod area $A_{r}$. Smaller rod sizes develop larger force output asymmetry. Increasing the rod size impacts the hysteresis between the force and velocity (see Figs. $4 \mathrm{a}$ and $4 \mathrm{c}$ and 5 ) and rotates the damping force ellipses into the first quadrant of the force-displacement plane due to the gas force. The hysteresis is larger when in compression than in rebound. Also, it can be shown that the gas force change magnitude is directly related to the rod area. Next, Fig. 6 reveals the pressures in each chamber of the damper vs. piston displacement and velocity. Note that the rebound chamber pressure dominates regardless of the damper operating conditions, i.e. it is clear that when the damper is in rebound the pressure in the lower chamber drops below gas pressure. Check valve (3) in the base valve opens, and there is flow through check valve (3) from the reservoir and into the compression chamber. In compression, the check valve in the piston opens and there is flow from the compression chamber into the rebound one. The effect of frequency manifested by an increase in the hysteresis in the force-velocity loops and force oscillations are shown in Fig. 7.

\section{Conclusions}

The author has analysed a novel model of a twin-tube MR damper concept. The study shows numerical results, however, the MR valve model is based on a verified bi-plastic theory and against real data which allows one to analyze the results with confidence (Gołdasz and Sapiński, 2012, 2013). Apart from the MR valve, the damper utilizes two one-way check valves in the piston and the base valve, respectively. The check valves offer extra means of tuning the output force in off-state conditions; this aspect of the concept is beyond the scope of this paper. Additionally, by using the check valves at the piston and the base of the damper, the flow through the piston is always in the same direction. To the author's knowledge no such model has been developed so far. As opposed to present MR structures, this configuration is asymmetric rebound-to-compression; the asymmetry is related to the rod size. To conclude, larger rod sizes minimize the asymmetry at the cost of rebound forces. The damper is more complex than single-tube structures but any performance and cost benefits, namely, lower friction, less stringent cylinder surface finish, may favour its applications. The damper works at a lower gas pressure than other MR damper structures, too. The twin-tube damper model can be a useful tool in various studies. The model relies on the information extracted mainly from engineering drawings and fluid data, which makes it suitable for fast sizing studies early in the design development stage. Transient studies through the $B$ - $\tau_{0}$ coupling are possible, too. 


\section{References}

1. De Carbon Ch., 1952, Shock absorbers, US Patent No. 2774446

2. Dimock G.A., Yoo J.-H., Wereley N.M., 2002, Quasi-steady Bingham biplastic analysis of electrorheological and magnetorheological dampers, Journal of Intelligent Material Systems and Structures, 13, 9, 549-559

3. GoŁdasz J., SAPiński, B., 2012, Nondimensional characterization of flow-mode magnetorheological fluid dampers, Journal of Intelligent Material Systems and Structures, 23, 14, 1545-1562

4. GoŁdasz J., SApiński B., 2013, Verification of magnetorheological shock absorber models with various piston configurations, Journal of Intelligent Material Systems and Structures, 24, 15, 1846-1864

5. Hong S.R., Gang W., Hu W., Wereley N.M., 2006, Liquid spring shock absorber with controllable magnetorheological damping, Proceedings of the Institution of Mechanical Engineers. Part D: Journal of Automotive Engineering, 220, 1019-1029

6. Jensen E., Oliver M.L., Kruckemeyer W.C., 2001, Twin-tube magnetorheological damper, US Patent Application No. 20020139624 A1

7. LANG H., 1977, A study of the characteristics of automotive hydraulic dampers at high stroking frequencies, Ph.D. Thesis, University of Michigan, US

8. LEE L., 1997, Numerical modeling for the hydraulic performance prediction of automotive monotube dampers, Vehicle System Dynamics, 28, 25-39

9. Manring N.D., 2005, Hydraulic Control Systems, John Wiley and Sons, New York

10. Mollica M., 1997, Nonlinear dynamic model and simulation of a high pressure monotube shock absorber using the bond graph method, M.Sc. Thesis, MIT, US

11. OAkley R., 2006, Twin-tube magnetorheological damper, European Patent Application No. 1908985 A1

12. Poynor J.C., 2001, Innovative designs for magnetorheological dampers, M.Sc. Thesis, Virginia Polytechnic Institute and State University, US

13. Segel L., LAng H., 1981, The mechanics of automotive hydraulic dampers at high stroking frequencies, Vehicle System Dynamics, 10, 2, 82-85 